\title{
MEDIA AUDIO-VISUAL SEMITA ANGGEN NINCAPANG KEWAGEDAN NYOLAHANG DRAMA BALI MODEREN RING SISIA KELAS VIII A SMP N 3 SINGARAJA
}

\author{
I Gede Ngurah Suwardhi Sesana ${ }^{1}$, I.B. Putra Manik Aryana ${ }^{1}$, I.B Made Ludy \\ Paryatna ${ }^{2}$ \\ Jurusan Pendidikan Bahasa Bali \\ Universitas Pendidikan Ganesha \\ Singaraja, Indonesia
E-mail : \{ngurahsuwardhi@gmail.com,manik.aryana, idabagusludy@gmail.com\}@undiksha.ac.id

\begin{abstract}
KUUB
Tetilikan puniki matetujon nelatarang (1) sapunapi tata cara guru ngemargiang peplajahan nganggen media audio-visual semita anggen nincapang kewagedan nyolahang drama Bali moderen, (2) napike prasida media audio-visual semita anggen nincapang kewagedan nyolahang drama Bali moderen , lan (3) sapunapi penampen sisia kelas VIII A SMP Negeri 3 sajeroning ngemargiang media audiovisual semita anggen nincapang kewagedan nyolahang drama Bali moderen. Jejering tetilik inggih punika sisia kelas VIII A, nglantur penandang tetilik inggih punika peplajahan nganggen piranti audiovisual semita lan kewagedan nyolahang drama Bali moderen. Kramaning mupulang data inggih punika kramaning seseleh, tes, lan angket/kuisioner. Pikolih sajeroning tetilikan puniki inggih punika (1) tata cara guru ngajahin nganggen media audio-visual semita sampun nganutin parindikan pengajahan manut ring RPP sane kakaryanin, (2) media audio-visual semita prasida nincapang kewagedan sisia nyolahang drama Bali moderen, nika kacingakin saking panincapan ketuntasan klasikal ring pailehan I inggih punika $68,57 \%$ dados $100 \%$ ring pailehan II. (3) sisia mapanampen becik indik media pengajahan audio-visual semita, nika kacingakin saking rerata penampen sisia ring pailehan I inggih puika 45,06 nincap ring pailehan II dados 46,23. Tetilikan sane kamargiang puniki nganggen data deskriptif kualitatif lan deskriptif kuantitatif.
\end{abstract}

Kruna jejaton: media audio-visual, Semita, Drama Bali Moderen

\begin{abstract}
ABSTRAK
Penelitian ini bertujuan untuk menjelaskan (1) bagaimana teknik guru mengajar menggunakan media audio-visual semita untuk meningkatkan kemampuan mendramatiskan drama Bali moderen, (2) apakah media audio-visual semita bisa meningkatkan kemampuan mendramatiskan drama Bali moderen, (3) bagaimana pendapat siswa kelas VIII A dalam proses pembelajaran drama Bali moderen menggunakan media audio-visual semita. Subjek penelitian ini adalah siswa kelas VIII A SMP N 3 Singaraja, dan objek penelitian ini adalah proses pembelajaran menggunakan media audiovisual semita serta kemampuan siswa mendramatiskan drama Bali moderen. Ada tiga tahap yang dilakukan dalam metode pengumpulan data, antara lain metode observasi, tes dan angket/kuisioner. Hasil dalam penelitian ini menunjukkan bahwa: (1) teknik guru mengajar menggunakan media audiovisual semita sudah sesuai dengan rencana pelaksanaan pembelajaran yang sudah dibuat sebelumnya. (2) media audio-visual semita dapat meningkatkan kemampuan medramatiskan drama Bali moderen, hal ini bisa dilihat dari peningkatan ketuntasan klasikal pada siklus I yaitu $68,57 \%$ menjadi $100 \%$ pada siklus II. (3) siswa memberikan respon positif terhadap proses pembelajaran menggunakan media audio-visual semita, hal inii terlihat dari peningkatan skor rerata pada siklus I
\end{abstract}


yaitu 45,06 dan 46,23 pada siklus II. Penelitian yang dilaksanakan ini menggunakan metode pengumpulan data deskriptif kualitatif dan deskriptif kuantitatif.

Kata kunci: media audio-visual, semita (ekspresi), drama Bali moderen.

\begin{abstract}
This study was aimed at describing (1) how is the teaching technique in using audio-visual semita media to improve the ability to dramatize Balinese modern drama, (2) whether audio-visual semita media is able to improve the ability to dramatize Balinese modern drama, and (3) what are students' responses on the use of audio-visual semita media in Balinese modern drama learning process. The subjects of this study were the students of VIII A class in SMP N 3 Singaraja and the objects of the study were the learning process using audio-visual media semita as well as the students' ability in dramatizing Balinese modern drama. There were 3 steps done in collecting the data intended, namely: observation method, test method, and questionnaire method. The result of this study showed that: (1) Teacher's technique in using audio-visual semita media was in accordance with the previous lesson plan designed before. (2) audio- visual semita media was able to improve the ability to dramatize Balinese modern drama, it can be seen from the improvement of classical exhaustiveness in the first cycle from $68.57 \%$ into $100 \%$ in the second cycle. (3) The students showed positive response toward the learning process using audio- visual semita media, it was seen from the improvement of mean score from 45.06 in the first cycle into 46.23 in the second cycle. This study was designed in the form of descriptive qualitative study as well as descriptive quantitative.
\end{abstract}

Key words: Audio-visual media, semita, Balinese modern drama

\section{PURWAKA}

Peplajahan ring kelas prasida kabaos mikolihang asil malajah sane becik, ri kala wenten piranti peplajahan sane prasida nyangkepin pamargin peplajahan punika. Silih sinunggil piranti sane prasida nyangkepin pamargin palajahan ring kelas inggih punika kawagedan guru sajeroning ngicenin peplajahan sane prasida karesepang olih para sisia. Kawagedan guru punika mabuat pisan antuk nyangkepin pamargin palajahan, krana yening guru nenten mrasidayang ngamargiang palajahan sane ngawinang para sisia meled sajeroning nyarengin pamargin palajahan sane kamargiang, dadosnyane para sisia nenten mrasidayang nguratiang lan ngaresepang peplajahan sane kamargiang ring kelas. Nika mawinan guru mangda nganggen kramaning (metode) sane patut mangda para sisia prasida aktif lan kreatif ri kala nyarengin pamargin peplajahan. Akeh piranti peplajahan sane prasida kaanggen olih guru, mangda pamargin peplajahan punika prasida mamargi becik lan prasida nincapang kayun para sisia malajah ring kelas. Silih sinunggil piranti peplajahan sane kaanggen inggih punika media audio-visual. Media audio-visual inggih punika piranti sane madaging suara lan gegambaran, minakadi rekaman video, film, slide suara, miwah sane lianan (Arsyad, 2012:148). Panilik nganggen media audio-visual semita, mangda nincapang kewagedan nyolahang drama Bali moderen. Media audio-visual semita punika becik pisan kaanggen ring peplajahan drama Bali moderen, santukan prasida nincapang imajinasi lan ngicen kaweruhan ring sisia ngenenin parindikan sane kapirengang lan kacingak ring video klip semita punika.

Video semita sane kaanggen inggih punika drama bawak sane mamurda "Terus Terang" olih SMP Negeri 1 Bangli, video klip lagu Bali sane mamurda "Jurus Andalan" sane katembangang olih Mang Nanik lan 20 semita ring muan manusa. Video punika kadadosang unteng pengajahan lan mangda sisia punika elah ngrereh semita ri kala nyolahang silih sinunggil pragina. Palajahan drama mabasa Bali ring sekolah madue pikenoh sane ageng pisan, santukan parasisia prasida malajahang raga indik 
kawentenan basa Bali ring kauripannyane. Drama prasida kadadosang sesuluh santukan drama pinaka gegambaran kauripan dados krama Bali. Sujatinnyane drama punika karya sastra sane madue daya rangsang, cipta, rasa,lan karsa sane pinih mautama (Endraswara, 2012). Drama embas saking pikayun ring kauripan manusa, punika mawinan drama nenten lempas saking lelintihan kauripan sarahina. Manut ring kawentenannyane, drama ngutamayang bebaosan (dialog), punika sane minayang drama saking fiksi sane tioasan. Dialog marupa bebaosan pantaraning pragina majeng ring pragina sane lianan. Kawentenan bebaosan ring drama banget mapikenoh, dadosnyane yening nenten madaging bebaosan, karya sastra punika nenten prasida kabaosang drama. Ahmadi (ring Endraswara, 2012: 11) maosang manut ring etimologi, drama mawit saking kruna 'drama' basa Greek (Yunani Kuno) drau sane maartos masolah (action) utawi maparilaksana sane manut ring pakem pragina soangsoang. Lianan ring ngutamayang bebaosan, drama taler madaging wiraga, wirama, lan wirasa. Wiraga inggih punika solah utawi parilaksana sane nginutin pakem pragina soang-soang manut ring lelintihan drama. Wirama inggih punika suara utawi bebaosan sane prasida kamedalang olih piranti suara manut ring bebaosan soang-soang pragina. Punika taler wirasa sapunapi pangrasa sane nginutin pahan drama sekadi bebaosan utawi monolog. Kewagedan sisia nyolahang drama mangda mrasidayang nglangkungin Kriteria Ketuntasan Minimal (KKM) 76. Ring aab sekadi mangkin sane kasengguh aab kaliyuga,sastra moderen sampun akeh kauningin, dadosnyane sisia nenten urati tekening plajahan basa Bali. antuk punika sepatutnyane guru mangda madue tata cara pengajahan sane anyar, sekadi media audio-visual puniki.

$$
\text { Bantang pikobet tetilik: }
$$

Sapunapi tatacara ngemargiang media audio-visual semita anggen nincapang kewagedan nyolahang drama Bali moderen ring sisia kelas VIII A SMP Negeri 3 Singaraja? (2) Napike prasida media audio-visual semita anggen nincapang kewagedan nyolahang drama
Bali moderen ring sisia kelas VIII A SMP Negeri 3 Singaraja? (3) Sapunapi penampen sisia kelas VIII A SMP Negeri 3 sajeroning ngemargiang media audiovisual semita anggen nincapang kewagedan nyolahang drama Bali moderen ring sisia kelas VIII A SMP Negeri 3 Singaraja?

Tetujon tetilik (1) Sumangdane prasida nelatarang indik tatacara ngemargiang media audio-visual semita anggen nincapang kewagedan nyolahang drama Bali moderen ring sisia kelas VIII A SMP Negeri 3 Singaraja. (1) Sumangdane prasida nelatarang indik media audiovisual semita prasida anggen nincapang kewagedan nyolahang drama Bali moderen ring sisia kelas VIII A SMP Negeri 3 Singaraja. (3) Sumangdane prasida nelatarang penampen sisia kelas VIII A SMP Negeri 3 Singaraja rikala ngemargiang media audio-visual semita anggen nincapang kewagedan nyolahang drama Bali moderen ring sisia kelas VIII A SMP Negeri 3 Singaraja.

Kawigunan Pamucuk, (1) Tetilikan puniki kaaptiang sumangdane ngicen kawigunan sajeroning ngalimbakang teori ngawigunaang media audio-visual ring palajahan, mangda tatujon malajah prasida sayan nincap. (2) Tetilikan puniki kaaptiang mawiguna majeng ring panglimbak ilmu pendidikan, cutetnyane ring palajahan basa Bali.

Kawigunan panglimbak. (1) Majeng ring sisia, ring tetilikan puniki para sisia pacang prasida mikolihang pengalaman malajah sane mawiguna, punika taler kaweruhan sisia ring sajeroning nyolahang drama Bali modern nincap. (2) Majeng ring guru, sajeroning tetilikan puniki kaaptiang prasida dados informasi sane banget mawiguna majeng ring guru rikanjekan ngalaksanayang makudangkudang utsaha anggen nincapang kaweruhan nyolahang utawi nyolahang drama sane ngawigunaang media audiovisual, cutetnyane ring palajahan basa Bali. (3) Majeng ring sekolah, tetilikan puniki kaaptiang prasida ngicen gatra sane mawiguna majeng manggala sekolah anggen ngambil kebijakan sane patut ring sajeroning utsaha ngicen tatacara palajahan sane patut ring 
sekolah. (4) Majeng ring penyeleh sane tiosan, tetilikan puniki taler kaaptiang mangda mawiguna majeng ring panilik sane lianan, kaanggen dasar ri kala nglaksanayang tetilikan sane manut lan kaanggen pratiwimba sareng tetilikan puniki mangda prasida ngamedalang tetilikan sane tiosan.

Suharto (ring Endraswara: 2011) maosang, semita (ekspresi) inggih punika mimik muka utawi kesan wajah. Semita masih kabaos nguncarang pikayun sane marupa tatujon, pikenoh lan emosi sane kasemitaang olih pasikian jadma. Suroto (1989: 134) maosang drama inggih punika makudang-kudang parindikan sane marupa konflik ring kauripan manusa sane madaging lelintihan satua lan prasida kasolahang ring ajeng pamiarsa. Cutetnyane drama ngutamayang lelintihan satua sane kawangun antuk dialog sane pacang kasolahang. Wenten makudangkudang sarana sane ngwangun drama minakadi: unteng lan tetuek, lelintihan, pawatekan, bebaosan (dialog), genah lan kasatuayang. Suroto (1989:76) minayang drama dados kekalih inggih punika drama tradisional lan drama moderen. Drama tradisional kabaos drama asli, santukan drama embas lan ngurip ring paguyuban krama Bali. Drama moderen inggih punika drama sane keni uparengga teater barat. Matiosan sareng drama tradisional sane sampun wenten ring kauripan parajana. Tata wangun drama manut Endraswara (2012:21) wenten babak, adegan, bebaosan, prolog lan epilog.

Nyolahang drama inggih punika pidabdab sane nginutin wirama lan wiraga mangda madue wirasa ring sajeroning drama. Wirasa sane becik pacang kapolihang olih sang pragina yening pragina punika sampun prasida mikayunin sapunapi parilaksanane sane patut nginutin naskah. Wenten makudangkudang tatacara sane prasida kalaksanayang rikala malajah tetikes inggih punika (1) nglatih angga, (2) nglatih suara, (3) nglatih observasi lan pikenoh (imajinasi), (4) nglatih teknik, (5) nglatih tetikes (akting) lan (6) nglimbakang skill ring latihan. Ring sajeroning drama nenten wenten tata cara nyolahang drama sane baku. Pragina kaaptiang mangda prasida nemuang teknik sane becik sane prasida nudut kayun sang sane miarsayang. Endraswara (2012: 72) maosang wenten pitu tata cara nyolahang drama sane kadadosang dasar anggen ngawantu rikala malajah drama. Makapitu tata cara punika minakadi (1) tata cara papeson, (2) tata cara ngicenin daging, (3) tata cara nglimbakang, (4) tata cara ngardi klimaks, (5) banban alon (tempo) lan irama, (6) mirengang lan nampenin, (7) tata cara mabebaosan. Manut Arsyad (2010: 3) kruna media wit saking basa latin "medius" sane maartos 'tengah', 'perantara', utawi 'pengantar'. Manut Gerlach lan Ely maosang media punika sampun ngeninin manusa, materi lan parisolah sane ngranayang sisia prasida ngamolihang aji pangweruhan. Ring sekolah sane dados media minakadi : guru, buku palajahan lan lingkungan utawi genah sisia ri kala nglaksanayang parikrama sareng sawitranyanne. Munadi (2013: 98) nyihnayang wenten 4 soroh media inggih punika (1) media suara (audio), (2) media gegambaran (visual), (3) media suara-gegambaran (audiovisual) lan (4) multimedia. Media audiovisual pinaka pasikian saking media audio sareng media visual. Ring media puniki nenten wantah mirengang suara nganggen indera pendengaran kemanten nanging, ring media puniki prasida kacingakin kawentenan gegambaran sane madaging ring media puniki. Sane ngaranjing ring media audio-visual inggih punika video, televisi, film lan komputer multimedia.

\section{KRAMANING TETILIK}

Tetilik puniki ngangge tetilik deskriptif kualitatif lan kuantitaif. Tetilik deskriptif kualitatif nganggen lan ngandelang data sane masifat non verbal (tertulis) lan tetilikan deskriptif kuantitatif nganggen data sane masifat verbal (angka). Ring sajeroning kramaning tetilik manut Wendra (2014: 52) prasida kawedarang indik, 1) Palihan Tetilik, palihan tetilik sane kaanggen ring tetilik puniki inggih punika penelitian tindakan kelas (PTK), 2) Jejering lan Panandang 
tetilik, sane dados jejering tetilik inggih punika sisia kelas VIII A SMP Negeri 3 Singaraja lan sane dados panandang ring tetilikan puniki nyolahang drama Bali moderen sane ngawigunayang media audio-visual semita, 3) pidabdab tetilik ngeninin indik pailehan sane jagi klaksanayang sajeroning nglaksanayang tetilikanPTK, 4) pangrencana nginutin pidabdab sane pacang kalaksanayang inggih punika (a) Makarya rencana pelaksanaan pembelajaran (RPP) nginutin palajahan sane pacang kasobyahang sajeroning asiki patemon, (b) Nyiagayang media audio-visualsane marupa video drama Bali moderen anggen nyobyahang palajahan, (c) Nyiagayang naskah drama Bali modern lan (d) Nyiagayang instrument anggen mupulang data sane kaperluang minakadi lembar observasi lan lembar tes anggen ngicen nilai parilaksana ring kelas ngeninin indik nyolahang drama Bali moderen ring sajeroning drama mabasa Bali, 5) paletan laksana Ring pailehan I, kalaksanayang kalih (2) patemon manut ring pangrencana sane sampun kakaryanang, 6) Seseleh (observasi)/pemastika kalaksanayang ri kala nglaksanayang paplajahan. Sumangdane mikolihang data selantur ipun kalaksanayang panureksan (pengamatan) salami paplajahan kawentenang, tatujonnyane mangda polih panampen sisia sajeroning pangajahan punika. Pidabdab sane pacang kalaksanayang sajeroning seseleh puniki sekadi (a) Nilikin parikrama (aktivitas) guru ri kala nelatarang paplajahan, (b) Nilikin parikrama sisia ri kala nyarengin paplajahan, (c) Nilikin tata cara pengajahan sane kalaksanayang olih guru ri kala ngamargiang pengajahan (d) Nilikin sisia ri kala nglaksanayang nyolahang drama Bali modern, 7) Mulat Wali, Mulat wali utawi refleksi inggih punika ngelingang lan minehang mawali ngenenin panglaksana sane pateh sakadi sane sampun kacatat ritatkala ngalaksanayang seseleh, 8) Mupulang data, Kramaning kanggen mupulang data inggih punika kramaning pratiaksa, tes, miwah angket; 9) Piranti Tetilik, piranti sane kaanggen sajeroning kramaning praktiaksa inggih punika lembar praktiaksa, piranti sane kaanggen sajeroning kramaning tes inggih punika tes lan piranti sane kaanggen sajeroning kramaning kuesioner/angket inggih punika pedoman kuesioner/angket. 10) Tata Cara Data Tureksa lan Sepat Sida. Data tureksa inggih punika pakaryan ngatur, ngurutang, lan nyekaang, ngicenin tanda utawi kode, lan ngategoriang data. Data pikolih plajahan prasida kapolihang saking tes unjuk kerja pinaka cihna kewagedan sisia nyolahang drama Bali moderen. Wiadin data penampen sisia kapolihang saking kuisioner.

\section{PIKOLIH TETILIKAN}

Ring pikolih puniki pacang katelatarang indik pikolih miwah tetepasan tetilikan, sane kakawitin olih panilik ring kelas VIII A SMP Negeri 3 Singaraja. Ring pikolih tetilikan punika pacang kawedarang indik data-data saking pikolih pratiaksa, tes, miwah angket/kuisioner. Yening ring tetepasan, punika pacang kawedarang ngenenin indik pikolih data utawi temuan bermakna ring tetilikan punika. Data sane sampun kapolihang punika pacang katureksaian, kawedarang lan katelatarang nganggen angka, tabel miwah lengkara-lengkara sane lengut turmaning prasida karesepang.

Ri kala nglaksanayang tetilikan puniki, panilik jagi nglaksanayang tetilikan tindakan kelas. Panilik nglaksanayang pratiaksa ring kelas VIII A SMP Negeri 3 Singaraja, ring tetilikan puniki panilik dados guru pengajahan sane kadadosang panglaksana tindakan miwah guru nglaksanayang sareng sawitran panilik nglaksanayang seseleh.

Patemon Kapertama kalaksanayang ring rahina Sukra tanggal 17 Maret 2017 galah palajahan ka 4-5 (15.25-16-10). Guru, panilik lan penyeleh ngranjing ka kelas VIII A SMP N 3 Singaraja. Ketua kelas ngenter sisia sane lianan ngaturang panganjali umat "Om Swastyastu". Guru, panilik, lan penyeleh raris ngwales panganjali punika. Sadurung ka materi peplajahan, sane kapertama guru nguningayang kapining para sisia kelas VIII A ngeninin indik rahinane mangkin galah peplajahan jagi kaicenin olih 
mahasisia sane jagi nglaksanayang tetilikan ring kelase puniki. Nglantur wusan punika, guru nyukserahang galah majeng ring panilik antuk nguningayang angga. Panilik lan penyeleh nguningayang angga majeng ring para sisia raris mahbahang napi tetujon ngranjing ka kelas majeng ring sisia. Sampun usan matur-atur majeng ring para sisia raris samian galah peplajahan kasuksrahang majeng ring guru pengajian ngraris, Panilik lan sawitran panilik ngrereh genah ring ungkur kelas. Selanturnyane, guru macak sisia sadurung ngawitin peplajahan, mangda guru uning wastan para sisia miwah akeh sisia sane rauh ring rahina mangkin. Wusan macak sisia, guru nglanturang antuk mungkah peplajahan antuk mahbahang cihna sane ngawinang labda (indikator), tatujon lan parikrama palajahan sane jagi kalaksanayang. Guru nguningayang indik peplajahan ring rahina mangkin ngeninin indik drama Bali moderen.

Ring undagan sane kapertama puniki guru kirang nyikakin para sisia sampun sayaga napike durung nyarengin pamargin peplajahan ring rahina mangkin (apersepsi). Sadurung ka undagan peplajahan sane kaping kalih inggih punika kegiatan inti, guru nguningayang majeng ring sisia mangda mirengang lan nyatet daging sane penting sajeroning materi sane jagi kamargiang.

Pamargin peplajahan kalanturang ka undagan sane kaping kalih inggih punika eksplorasi, elaborasi lan konfirmasi. Guru ngawitin nelatarang materi antuk ngicenin pitaken majeng ring sisia ngeninin indik teges drama. Guru ngicenin para sisia galah nyawis pitaken punika. Wusan punika guru nguningayang majeng ring sisia, sira sane prasida nyawis nglantur, wenten makudang sisia sane ngunggahang tangan lan nawis pitaken punika, nanging cawisan saking para sisia durung prasida kabaos jangkep. Mangda nenten akeh nelasang galah, salanturnyane guru sane nelatarang ngeninin indik teges drama, para sisia nguratiang napi sane katelatarang olih guru lan nyatet napi sane penting ring peplajahan punika.
Wusan guru nelatarang teges drama, nglantur guru nelatarang indik sampunnapi tata cara masemita sane becik ri kala nyolahang drama. Para sisia sampun kacingak nenten urati olih guru. Naanin pikobet punika guru nguningang majeng ring sisia mangda urati kapining pamargin peplajahan ne mangkin. Wusan sisia sampun kacingak urati olih guru, nglantur guru nglanturang nelatarang ngeninin indik semita sane becik. $\mathrm{Ri}$ sampune puput nelatarang materi sane kamargiang, guru nganggen media audiovisual semita antuk ngicenin wewehan ring materi semita. Ring piranti audiovisual punika ka tayangang sesolahan drama bawak sane mamurda "Terus Terang" sane kasolahang olih sisia SMP N 1 Banggli. Nglantur guru mituduhin para sisia miarsayang lan nyingakin semita sane kamargiang olih soang-soang pragina. Wusan miarsayang drama bawak punika, guru ngicenin pitaken majeng ring sisia ngeninin indik semita sane kasolahang olih soang-soang pragina napike wenten iwang utawi ten. Nglantur wenten makudang-kudang sisia sane ngunggahang tangan lan ngicenin penampen sane becik majeng ring semita sane kasolahang olih pragina ring media audio-visual. Para sisia kacingak saat pisan ngicenin wewehan lan penampen ngeninin indik semita sane kasolahang punika. Ring parikrama puniki, pamargin peplajahan kamargiang selami 70 menit.

Ring panguntat hasil peplajahan, guru nyutetang daging peplajahan sane sampun kamargiang. Sadurung muputang pamargin peplajahan, guru mituduhin sisia mangda ngawi sekaa akehnyane 4 sekaa. Wusan sisia ngawi sekaa, nglantur guru ngicenin naskah drama bawak kapining soang-soang sekaa lan, ring awuku sane jagi rauh jagi kaicenin tes nyolahang drama manut naskah sane sampun kaicen majeng ring soang-soang sekaa. Wusan punika pamargin peplajahan kapuputang antuk pama santih, sane kajudi olih ketua kelas.

Patemon sane kaping kalih kalaksanayang ring awuku selanturnyane inggih punika ring rahina Sukra tanggal 24 Maret 2017 galah palajahan ka 4-5 (15.25-16-10). Guru, panilik lan penyeleh 
ngranjing ka kelas VIII A SMP N 3 Singaraja. Ketua kelas ngenter sisia sane lianan ngaturang panganjali umat "Om Swastyastu". Guru, panilik, lan penyeleh raris ngwales panganjali punika. Wusan punika, panilik lan sawitran panilik ngrereh genah ring ungkur kelas. Wusan punika guru nguningang majeng ring sisia, ring patemon kekalih puniki nenten malih nglaksanayang peplajahan nanging nglaksanayang tes nyolahang drama manut naskah sane sampun kaicen majeng ring soang-soang sekaa. Sadurung ngawitin tes sane kapertama guru mituduhin sisia mangda negang sareng sekaa nyane nglantur, sekaa sane durung polih giliran nyingakin sekaa sane nyolahang drama ka arep kelas. Selanturnyane guru ngicenin nomor dada mangda guru aluh ngicenin biji majeng ring soang-soang sisia. Wusan punika guru mituduhin soang-soang sekaa nyolahang drama maut naskah drama sane sampun kaicen majeng ring soangsoang sekaa. Ring parikrama tes puniki, guru dados panureksa lan ngerereh genah ring ungkur kelas sareng para penyeleh. Samian sekaa meled pisan miarsayang lan tertib ri kala silih sinunggil sekaa nyolahang drama ka arep kelas. Tes kalaksanayang kirang langkung 60 menit.

Wusan tes kalaksanayang, guru mituduhin sisia mangda ngrereh genah soang-soang. Salanturnyane, guru ngicenin motivasi lan wewehan mangda nincapang malih kewagedan sajeroning nyolahang drama. Salanturnyane guru nakenin para sisia pikobet (kesulitan) napi sane kapolihang sajeroning nyolahang drama. Pikobet sane kapolihang olih sisia inggih punika ragu sajeroning masemita lan nu lek-lekan kapining para sawitra ri kala masamita sane patut. Mirengang pikobet punika guru ngicenin penampen anggen natasin pikobet punika.

$\mathrm{Ri}$ sampune puput ngicenin wewehan, guru ngicenin angket/kuisioner majeng ring soang-soang sisia nglantur, sisia ngicenin penampen manut lengkara sane sampun kasurat ring angket/kuisioner punika. Malih pisan guru mataken majeng ring sisia usan ngisinin angket/kuisioner.
Dwaning nenten wenten pitaken saking para sisia, guru lan para sisia nyutetang hasil peplajahan ring rahina mangkin. Guru ngicenin penghargaan majeng ring sekaa sane sampun waged lan trepti ri kala nyolahang drama antuk sesumbung lan koplokan tangan. Pamargin tes ring pailehan I puniki kapuputang antuk parama santih.

Pikolih nyolahang drama Bali moderen ring pailehan I ngenin inidik kewagedan sisia nyolahang drama Bali moderen ngamolihang rerata 78,89 lan ketuntasan Klasikal nyane inggih punika 68,57 . Nanging ring pikolih penampen sisia angket, sampun ngamolihang pikolih becik inggih punika akehnyane 27 $(77,14 \%)$ sisia antuk kategori pinih positif.

Mulat wali pailehan I punika minakadi kaanggen jalaran mangda plajahan memargi antar lan sayan ngamecikang. Kahanan sane wenten ring pailehan I punika pinaka pikobet sane pacang karereh, pamuput nyane wau raris kamargiang ring siklus selanturnyane. Saeroning nincapang rasa ngaat sisia nyolahang drama Bali moderen, guru mangda nincapang tata caranyane ngamargiang peplajahan utawil menyenangkan.

Patemon Kapertama kalaksanayang ring rahina Sukra tanggal 19 Mei 2017 galah palajahan ka 4-5 (15.25-16-10). Guru, panilik lan penyeleh ngranjing ka kelas VIII A SMP N 3 Singaraja. Ketua kelas ngenter sisia sane lianan ngaturang panganjali umat "Om Swastyastu". Guru, panilik, Ian sawitran panilik raris ngwales panganjali punika. Selanturnyane panilik lan sawitran panilik ngrereh genah ring ungkur kelas lan galah peplajahan kasuksrahang majeng ring guru prngajian. Selanturnyane, guru macak sisia sadurung ngawitin peplajahan, mangda guru uning wastan para sisia miwah akeh sisia sane rauh ring rahina mangkin. Wusan macak sisia, guru nglanturang antuk mungkah peplajahan antuk mahbahang cihna sane ngawinang labda (indikator), tatujon lan parikrama panajahan sane jagi kalaksanayang. Guru nguningayang indik peplajahan ring rahina mangkin ngeninin indik drama Bali moderen. 
Ring undagan sane kapertama puniki guru mataken majeng ring sisia, sampun sayaga nyarengin peplajahan ne mangkin?. Sisia nyawis sinarengan ngorahang sampun. Nglantur, guru ngicenin para sisia apersepsi sane maiketan sareng daging peplajahan sane jagi kamargiang. Wusan punika para sisia sampun kacingak saat pisan nyarengin pamagin peplajahanne mangkin. Ring pamungkah peplajahan puniki akehnyane nelasang galah kirang langkung 10 menit.

Pamargin peplajahan kalanturang ka undagan sane kaping kalih inggih punika eksplorasi, elaborasi lan konfirmasi. Guru ngawitin nelatarang materi antuk ngicenin pitaken majeng ring sisia ngeninin indik teges drama malih. Nika kalaksanayang mangda guru uning napikeh sisia sampun ngresep sareng teges drama sane kamargiang ring pailehan I. Guru ngicenin para sisia galah nyawis pitaken punika. Wusan punika guru nguningayang majeng ring sisia, sira sane prasida nyawis nglantur, wenten makudang sisia sane ngunggahang tangan lan nawis pitaken punika. Akeh sisia sane nyawis becik pisan lan jangkep ngeninin indik teges drama. Dwaning sampun sekadi asampunika guru nglanturang panelataran selanturnyane ngeninin indik tata cara masemita sane becik.

Para sisia sampun kacingak urati lan trepti ri kala nyarengin pamargin peplajahan punika. Wenten silih sinunggil sisia sane masemu ucem ri kala nyarengin peplajahan. Naanin pikobet punika guru maekin lan nakenang majeng ring sisia napi ngawinang asampunika. Wusan nakenin sisia, guru nglanturang nelatarang materi ring ajeng kelas lan nguningang majeng ring sisia mangda urati kapining pamargin peplajahan ne mangkin. Wusan sisia sampun kacingak urati olih guru, nglantur guru nglanturang nelatarang ngeninin indik semita sane becik. Ri sampune puput nelatarang materi sane kamargiang, guru nganggen media audio-visual semita antuk ngicenin wewehan ring materi semita. Ring media audio-visual punika kasobyahang lagu pop Bali sane mamurda "Jurus Andalan" sane katembangang olih Mang Nanik.
Guru nganggen audio visual lagu pop Bali, dwaning ri kala tembang punika mamargi wenten sesolahan sane nyihnayang semita ring tembang punika. Antuk punika guru nganggen audio-visual semita ring tembang pop Bali. Nglantur guru mituduhin para sisia miarsayang lan nyingakin semita sane kamargiang olih soang-soang pragina sane wenten ring tembang punika. Wusan miarsayang tembang punika, guru ngicenin pitaken majeng ring sisia ngeninin indik semita sane kasolahang olih soang-soang pragina napike wenten iwang utawi ten. Nglantur wenten makudang-kudang sisia sane ngunggahang tangan lan ngicenin penampen sane becik majeng ring semita sane kasolahang olih pragina ring media audio-visua semita punika. Nglantur wusan polih mabligbagan sareng sisia, malih pisan guru nyobyahang audiovisual sane mamurda "21 semita sane wenten ring mua manusa" sane video nyane kaambil ring youtube. Wusan miarsayang, guru mituduhin silih sinunggil sisia ka ajeng kelas, jagi nyolahang napi manten semita sane kapolihang saking video iwau. Para sisia kacingak saat pisan nyarengin pamargin peplajahan punika. Ring parikrama puniki, pamargin peplajahan kamargiang selami 70 menit.

Ring panguntat hasil peplajahan, guru nyutetang daging peplajahan sane sampun kamargiang. Sadurung muputang pamargin peplajahan, guru mituduhin sisia mangda ngawi sekaa sane anyar akehnyane 4 sekaa. Wusan sisia ngawi sekaa, nglantur guru ngicenin naskah drama bawak kapining soang-soang sekaa lan, ring awuku sane jagi rauh jagi kaicenin tes nyolahang drama manut naskah sane sampun kaicen majeng ring soang-soang sekaa. Dwaning nenten wenten pikobet utawi pitaken saking sisia, pamargin peplajahan kapuputang antuk pama santih, sane kajudi olih ketua kelas.

Patemon sane kaping kalih ring pailehan II kalaksanayang ring awuku selanturnyane inggih punika ring rahina Sukra tanggal 26 Mei 2017 galah palajahan ka 4-5 (15.25-16-10). Guru, panilik lan penyeleh ngranjing ka kelas VIII A SMP N 3 Singaraja. Ketua kelas ngenter sisia sane lianan ngaturang 
panganjali umat "Om Swastyastu". Guru, panilik, lan penyeleh raris ngwales panganjali punika. Wusan punika, panilik lan sawitran panilik ngrereh genah ring ungkur kelas. Wusan punika guru nguningang majeng ring sisia, ring patemon kekalih puniki nenten malih nglaksanayang peplajahan nanging nglaksanayang tes nyolahang drama manut naskah sane sampun kaicen majeng ring soang-soang sekaa. Sadurung ngawitin tes sane kapertama guru macak sisia lan makasami sisia rauh utawi nihil. Wusan punika, guru nguningayang indikator palajahan, makadadosnyane sisia prasida nyiagayang pidabdab nyolahang drama Bali modern. Nglantur, Guru ngicen galah mangda soang-soang sekaa mataki-taki jagi nyolahang drama sane sampun kapituduhin.

Ri sampun sisia kacingak sayaga antuk ngamargiang tes, guru mituduhin sekaa sane durung polih giliran nyingakin sekaa sane nyolahang drama ka arep kelas. Selanturnyane guru ngicenin nomor dada mangda guru aluh ngicenin biji majeng ring soang-soang sisia. Wusan punika guru mituduhin soang-soang sekaa nyolahang drama maut naskah drama sane sampun kaicen majeng ring soangsoang sekaa. Ring parikrama tes puniki, guru dados panureksa lan ngerereh genah ring ungkur kelas sareng para penyeleh. Samian sekaa meled pisan miarsayang lan trepti ri kala silih sinunggil sekaa nyolahang drama ka arep kelas. Tes kalaksanayang kirang langkung 60 menit.

Wusan tes kalaksanayang, guru mituduhin sisia mangda ngicenin penampen ngeninin indik media-audio visual semita sane kaanggen piranti ngajahin ring kelas. Para sisia nguningang malajah nganggn media audio-visual semita, prasida ngawinang manah para sisia seneng lan akeh semita sane prasida kadulad saking video sane kamargiang. Para sisia merasa seneng rikala manahne nenten becik wenten video sane kamargiang sane prasida ngawinang rasa meled malajah majeng ring sisia. $\mathrm{Ri}$ sampune puput mabligbagan sareng sisia, guru ngicenin angket/kuisioner majeng ring soang-soang sisia nglantur, sisia ngicenin penampen manut lengkara sane sampun kasurat ring angket/kuisioner punika. Malih pisan guru mataken majeng ring sisia usan ngisinin angket/kuisioner.

Dwaning nenten wenten pitaken saking para sisia, guru lan para sisia nyutetang hasil peplajahan ring rahina mangkin. Guru ngicenin penghargaan majeng ring sekaa sane sampun waged lan trepti ri kala nyolahang drama antuk pujian lan koplokan tangan. Pamargin tes ring pailehan I puniki kapuputang antuk parama santih.

Pikolih nyolahang drama Bali moderen ring pailehan II ngenin inidik kewagedan sisia nyolahang drama Bali moderen ngamolihang rerata 82,14 nglantur ketuntasan klasikalnyane inggih punika $100 \%$. Salanturnyane ring pikolih penampen sisia angket, sampun ngamolihang pikolih becik inggih punika akehnyane 33 sisia $(94,29 \%)$ sisia antuk kategori pinih positif.

Saking asil sane sampun katelatarang ring arep, palaksana pailehan II sampun mamargi labda karya tur mapikolih becik. Pamikolihan asil ring pailehan II sampun prasida nglangkungin asil sane kalaksanayang ring pailehan I. Ring pailehan II, pamargin peplajahan sampun mamargi becik pisan, parikrama guru sare sisia sampun sesai kalaksanayang. Nenten wenten rasa kimud utawi jerih ri kala mataken sareng guru lan nyolahang drama ka arep kelas. Samian palaksana pailehan II punika sane ngawinang asil kewagedan nyolahang drama sisia prasida nincap.

Manut asil saking pailehan II, prasida katelatarang yening pamargin peplajahan nganggen media audio-visual semia prasida nincapang kewagedan nyolahang drama Bali moderen sisia kelaas VIII A SMP Negeri 3 Singaraja.

Perbandingan skor kewagedan nyolahang drama Bali moderen sane ngawigunayang media audio-visual semita anggen nincapang kewagedan nyolahang drama Bali moderen ring sisia kela VIII A SMP Negeri 3 Singaraja inggih punika sekadi ring sor. Pikolih melajah sisia ring siklus II punika sampun kabaos mapikolih, santukan sampun prasida nincapang 
kewagedan nyurat puisi Bali anyar sisia kelas VIII A SMP Negeri 3 Singaraja. Pikolih punika nganutin sepat sida inggih punika sisia sane ngamolihang biji $\geq 75$ akehnyane 35 diri sisia (100\%). Pikolih nyolahang drama Bali moderen ring pailehan I ngenin inidik kewagedan sisia nyolahang drama Bali moderen ngamolihang rerata 78,89 . Akehnyane 5 $(14,29 \%)$ sisia polih nilai sane pinih becik (PB), akehnyane 19 sisia $(54,28 \%)$ sisia polih nilai sane becik (B) lan sane kaping untat akehnyane $11 \quad(31,43 \%)$ sisia polih nilai sane sedeng (S).nglantur ring pikolih penampen sisia angket, sampun ngamolihang pikolih becik inggih punika akehnyane $27(77,14)$ antuk kategori pinih positif. Manut saking 35 sisia, sane polih nilai sane anut sareng kriteria ketuntasan minimal (KKM) wantah 20 (68,57\%) sisia. Nglantur sisia sane polih nilai durung anut sareng $K K M$ akehnyane 11 sisia (31,43\%). Manut kriteria ketuntasan sane katetepang ring bab III, media audio-visual semita anggen nincapang kewagedan nyolahang drama Bali moderen kabaos puput ri kala $75 \%$ sisia polih nilai sane anut sareng KKM. Antuk punika, pamargin tetilikan ring pailehan I durung kabaos berhasil dadosnyane durung prasida kapuputang. Nglantur pikolih nyolahang drama Bali moderen ring pailehan II ngenin inidik kewagedan sisia nyolahang drama Bali moderen ngamolihang rerata 82,14 . Akehnyane 7 sisia $(20,00 \%)$ polih niai pinih becik (PB) lan malih 28 sisia $(80,00 \%)$ polih nilai becik (B). Akeh sisia kelas VIII A SMP N 3 Singaraja, inggih punika 35 sisia (100\%) ngamolihang nilai sane anut sareng KKM inggih punika 76. Manut kriteria ketuntasan sane sampun katetepang ring bab III, peplajahan nganggen media audio-visual semita angen nincapang kewagedan nyolahang drama Bali moderen sampun prasida kapuputang yening $75 \%$ sisia polih nilai sane anut sareng KKM. Antuk punika pamargin tetilikan ring pailehan II puniki sampun kabaos puput (berhasil) sinah tetilikan puniki prasida kapuputang. Ring pikolih penampen sisia angket, sampun ngamolihang pikolih becik inggih punika akehnyane 34 sisia $(97,14)$ antuk kategori pinih positif.

\section{IMPLIKASI}

Sajeroning tetilikan puniki, ring kawigunannyane dumogi prasida kaanggen uger-uger ri kala guru ngamargiang palajahan sane mapaiketan sareng peplajahan drama mabasa Bali. Salanturnyane sida nganggen metode puniki manut dudonan tata cara sane patut sajeoning nganggen uger-uger utawi tata cara ri kala ngajahin nganggen media audio-visual mangda sisiane prasida ngresepang napi sane kaplajahin manut pelajahan punika.Yening materi indik pelajahan drama mabasa Bali kesarengin piranti sane manut, sisia dangan ngaresepang materi sane katelatarang, sida nincapang kewagedan sisia, kaweruhan sisia, uratian sisia, miwah sane tiosan. Sane ngawinang niki sida nincap sampun mabukti ring tetilikan puniki inggih punika sampun nincap kewagedan para sisiane nyolahang rdama Bali moderen nganggen media audiovisual lan sisiane taler akeh madue pikenoh (inspirasi) sajeroning nyolahang drama Bali moderen.

Malarapan antuk kawigunan tetilikan ring kewagedan sisia ri kala nyolahang drama Bali moderen punika banget mabuat pisan kaanggen nincapang kewagedan sisia nyolahang drama Bali modern sane ngawigunayang piranti audio-visual semita puniki kacingak saking ketuntasan klasikal sane nincap dados $100 \%$ ring kelas VIII A SMP Negeri 3 Singaraja. Dumogi ring kelas sane tiosan prasida ngicen pemargi sane becik pateh sekadi tetilikan puniki sane kamargiang olih panilik mangkin. Nglantur prasida kaanggen imba ri kala ngamargiang tetilikan sane pateh ring tetilikan panilik puniki, inggih punika tetilikan PTK.

Malarapan antuk sinalih tunggil kawigunan tetilikan ring penampen sisia, ri kala nyarengin peplajahan nyolahang drama Bali moderen puniki prasida kacingak ring pamikolih sisia ngicen penampen sane pinih cumpu sareng pelajahan nyolahang drama Bali moderen sane ngawigunayang media audio-visual semita ngwantu nincapang kewagedan sisia nyolahang drama Bali moderen. 
Punika prasida ngicenin kontribusi sekadi sane kapanggihin ring tetilikan puniki mangda prasida mabuat anggen imba tetilikan olih panilik sane lianan.

\section{PAMUPUT}

Manut uraian sajeroning panelataran ring ajeng ngeninin indik peplajahan drama nganggen media audio-visual semita ring sisia kelas VIII A SMP Negeri 3 Singaraja, prasida kacutetang inggih punika : (1) Kawagedan sisia nyolahang drama Bali moderen punika, nenten lempas saking tata cara guru ngajahin. Indik punika kaarepang mangda guru prasida ngawigunayang piranti-piranti sane mabuat anggen nincapang kawegedan sisia nyolahang drama Bali moderen, sekadi media audio-visual semita sane panilik anggen tetilikan puniki. Guru kaaptiang nelebin sisia antuk ngawigunayang pepelihan kruna sane patut, lan rerambangan (maya cita), sumangdane drama sane kasolahang madue semita sane becik lan madue pabesen sane mawiguna. (2) media audio-visual semita prasida nincapang kewagedan nyolahang drama Bali moderen ring sisia kelas VIII A SMP Negeri 3 Singaraja. Indik punika prasida kacingak saking rata-rata pikolih kewagedan nyolahang drama Bali moderen sisia ring pailehan I inggih punika $78,89 \%$. Ring pailehan II, rata-rata nilai sisia inggih punika 82,14. Panincapan pikolih melajah sisia saking pailehan I $(78,89)$ ka pailehan II $(82,14)$ inggih punika 3,25 poin. Salanturnyane ketuntasan klasikal $(K K)$ sisia masih nincap saking pailehan I ka pailehan II. Ring pailehan I, $K K$ sane kapolihang inggih punika $68,57 \%$ lan nincap ka pailehan II inggih punika 100\%. (3) pikolih penampen sisia utawi angket sisia sampun ngamolihang penampen becik. Indik media audio-visual semita sane prasida nincapang kewagedan nyolahang drama Bali pailehan ring sisia kelas VIII A SMP Negeri 3 Singaraja. Pikolih nontes ring pailehan I mikolihang rerata inggih punika 45,06 sane akehnyane 27 $(77,14 \%)$ sisia ngicen penampen sane pinih positif lan siisia sane lianan inggih punika $8 \quad(22,86 \%)$ sisia ngicenin penampen sane positif. Nglantur nincap ring pikolih pailehan II inggih punika mikolihang rerata 46,23 sane nincap malih 1,17 biji. Akehnyane 33 (94,29\%) sisia ngicen penampen sane pinih positif lan sisia sane lianan wantah $2(5,71 \%)$ sisia ngicenin penampen sane positif Antuk punika penampen sisia kapining media audio-visual semita sampun anut sareng persyaratan minimal inggih punika $75 \%$ sisia ngicenin penampen sane pinih positif.

Manut pikolih ring ajeng, prasida kauningayang piteket-piteket sekadi (1) Kewagedan sisia nyolahang drama Bali moderen punika, nenten lempas saking tata cara guru ngajahin. Indik punika kaarepang mangda guru prasida ngawigunayang piranti-piranti sane mabuat anggen nincapang kewegedan sisia nyolahang drama Bali moderen, sekadi piranti audio-visual semita sane panilik anggen tetilikan puniki. (2) Sane ngawinang sisia punika nenten ngamolihang nilai nganutin sepat sida utawi $\leq 70$, indik punika nenten lempas saking praragan sisia punika sane nenten urati sareng parindikan guru ngajahin. Nika mawinan kaarepang sisia ri kala nyolahang drama mangda masemita sane anut sareng pagina sane kasolahang miwah madue pikenoh sane patut sadurung nglaksanayang tetikes.

\section{KAPUSTAKAAN}

Agustini, Luh Putu. 2013. Pemanfaatan Media Kartu Ekspresi untuk Meningkatkan Kemampuan Bermain Peran dalam Pembelajaran Drama Berbahasa Bali Siswa Kelas X TB 1 SMK Pariwisata Triatma Jaya Singaraja. Skripsi (tidak diterbitkan). Jurusan Pendidikan Bahasa Bali, FBS Undiksha.

Arsyad, Azhar.2010. Media Pembelajaran.Jakarta: Rajawali Press.

Endraswara, Suwardi. 2012. Metode Pembelajaran Drama (Presiasi, Ekspresi, dan Pengkajian). Yogyakarta: CAPS. 
Suroto. 1989. Teori dan Bimbingan Apresiasi Sastra Indonesia. Jakarta: Penerbit Erlangga.

Wendra, I Wayan. 2014. Buku Ajar Penulisan Karya IImiah. Singaraja: Undiksha. 